\title{
Innovative problem solving methods in education field
}

\author{
Nebojša Stefanović ${ }^{1}$, Radivoje Mitrović ${ }^{2}$ Predrag Popovic ${ }^{3, *}$ \\ ${ }^{1}$ Ministry of Education and Science, Belgrade, Serbia \\ ${ }^{2}$ Faculty of Mechanical Engineering in Belgrade, Belgrade University, Belgrade, Serbia \\ ${ }^{3}$ Vinca Institute of Nuclear Sciences, Belgrade University, Belgrade, Serbia
}

\section{Email address:}

biro@vinca.rs (P. Popovic)

\section{To cite this article:}

Nebojša Stefanović, Radivoje Mitrović, Predrag Popović. Innovative Problem Solving Methods in Education Field, Education Journal. Vol. 2, No. 2, 2013, pp. 27-35. doi: 10.11648/j.edu.20130202.12

\begin{abstract}
TRIZ (Теория Решеня Изобретательных Задач - Theory of innovative problem solving) - is the method enabling stimulation of creative problem solving by way of using the developed knowledge bases. It has been proved that innovative inventions from certain fields of technologies can be applied not only to technical but also to the social human activities, and thus to education as well. TRIZ exploration begins with the hypothesis that there are no universal principles of finding creative solution for any problem based on innovation and advanced technologies. TRIZ support has shown excellent results in the field of education, as well. The system of education is one of the most complex social systems, burdened with multiple contradictions. The education system's contradictions comprise wishes of parents versus capabilities of children, wishes of teachers versus educational institution capabilities, etc., so that the need arises for presenting the assembling of educational requirements of the society for improving the level of education, versus writing new educational plans, by way of improved technical resources of the society. Basic TRIZ concepts says that creative solutions require removing of contradictions. Being creative, the TRIZ method is an important part of the technological education program at high schools of professional studies and at faculties in the course of studying. An important link to TRIZ method application in education is envisaging of teachers' perceptions in the education technology, which is the first step towards introducing TRIZ method into the educational plan and program at lower educational levels as well. The paper offers and develops application of new models of education management by way of TRIZ method, by the matrix of forty innovative principles, as well as by the matrix of four most influential features, with the special contribution of applying creative principles in the field of education.
\end{abstract}

Keywords: Creative Methods, Contradictions, Education, TRIZ

\section{Introduction}

TRIZ represents a method of solving the problem of system safety, based on the set of data obtained from the base of empiric solutions and exploitation of a similar system. The method utilizes the principle of REPEATABILITY and it was developed by Altshuller [1], who determined that there was a lack of methods for creating new solutions. The TRIZ method today represents an international way of approaching creativity in resolving the problem of safety integration, which provides results based on discovering causes between problems and the search for solutions. The TRIZ method identifies 2 types of contradictions:

Physical contradictions, - these signify direct opposition towards two values for the same parameter which formulates the educational system.

Technical contradictions denote such situations where improvement of a parameter A leads towards worsening of parameter B.

Organizational contradictions occur in educational system as well. By resolving the organizational contradictions, we reach the situation to have to resolve anew the physical or technical contradictions. The TRIZ method proposes to avoid the exhaustion methods: - by using the contradiction matrixes in resolving the problem of technical contradictions, as well as by changes in the system, in resolving physical contradictions. In analyzing the complex picture and details of an event, TRIZ can have the multiple purposes to check creativity in the educational process and to check creative models. The basis of this method is founded on the following:

analysis of regulations in the field of education and

reports and experiences in the course of educational cycle. 
On the basis of the above stated, various solution structures are made in the process of planning and realizing the educational cycle, so that they need to be taken into consideration as soon as possible. The solutions obtained by the method of compromises in education show applicability of this method's principles. The contradictions matrix implies a set of educational system's features which, individually or in synergy, influence each other in the contradictions affecting productivity as the final measure of the educational system efficiency.

\section{TRIZ Method Application in Educa- tional System Analysis}

TRIZ integrated solutions should inspire, engage and motivate the usage of technological methods in resolving problems in education. Through its innovative modules, the TRIZ method looks for solutions by presenting the next generation of contemporary practice's "products", with the objective of broadening limits and raising standards in the sector of education. TRIZ solutions are expected to enable efficient automatization of all operating procedures and to provide accessibility and knowledge of all the educational process facts by ample information, thus providing a comprehensive counseling display on an integrated platform [2], [3]. Access to the process from any place at any time, represents its fully integrated environment. On the basis of a scientific theory, methods have been developed by studying educational institutions, applying various systems' development patterns, for searching for creative solutions. These methods involve three major components:

analysis of the logic nature of educational system and its problems,

application of specific knowledge data bases as the most efficient problem-solving method,

means for overcoming psychological inertia in the problem-solving process.

The basic ideas of TRIZ method implementation in pedagogy [4] are as follows:

study on any topic, such as system development,

relationships between different subjects through educational system evolution patterns,

application of the problem-solving creative method in lecturing to students,

creative problem-solving by thematic lecturing by demonstration models,

mastering the methods for overcoming psychological inertia.

In educational system, the TRIZ method application has got an additional group of goals:

preserving and strengthening child's innate creative disposition,

forming of creativity and of vital orientations towards accomplishing goals,

forming of creative way of thinking,

In order to put it briefly, due to the limited space for this paper, the TRIZ method basic characteristics are stated here, as follows:

Flexibility, quick response and fast reaction to the educational system changes,

Excellent design in the process implementation,

High level of rendered services in the process of educating,

Integrated solution of education management through one platform,

Reports adjusted to the educational system needs,

Easy and comprehensible user environment,

Simpler application as compared to other methods,

Higher number of options offered for application from higher management level.

TRIZ exploration starts with the hypothesis that there are no universal principles for finding a creative solution for any problem based on innovation and advanced technology. If these principles could be identified, task teams would have, in the process of designing, more creativity and they would be capable of predicting much more operative situations. TRIZ method is based on the following principles:

Someone there, somewhere, has got the solution for this problem or for a similar one.

Creativity today is based on finding a solution and adjusting it to the problem under consideration.

The principle of solution finding according to the TRIZ method is depicted in Figure 1. The grey filled arrows show the transformation during formulating one problem or a solution towards another problem. This process comprises the problem analysis and analytical usage of empiric data from the existing basis. The streaked arrow represents the analogue thinking in searching for solutions for the specific case. The crossed-out streaked arrow shows that the TRIZ method cannot be used in searching for a specific problem by a specific solution.

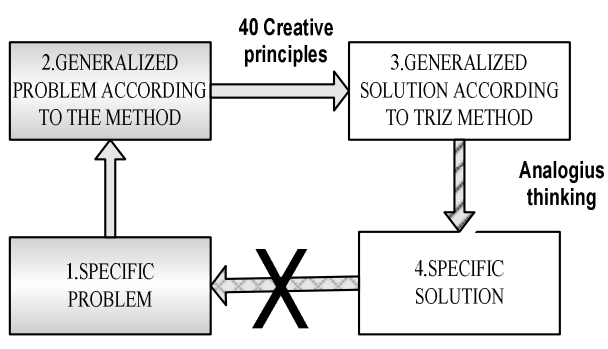

Figure 1. The solution finding principle according to TRIZ method.

The TRIZ method comprises five steps [5] forcing the user to overcome the existing psychological preconceived notions by a structured approach by a divergent way of thinking about the problem, which is typical in finding ideas for the current problem, as follows:

1. Problem determining and defining;

2. Re-formulating the problem in the sense of contradictions;

3. Formulating technical contradictions by using the 40-feature matrix; 
4. Search for an analogous solution out of the 40principle matrix;

5. Determining a final creative solution.

In developing the method, the knowledge has been arrived at that this method, in the process of planning the educational system behavior, has shown excellent performances regarding speed of analyzing and solution finding. Comparing the method to numerous other methods [4], the knowledge has been arrived at that decisions can be reached by TRIZ method on the basis of seven criteria, as follows:

1. Technique of conditioning/motivating/organizing;

2. Technique based on innovation knowledge;

3. Technique of systematizing;

4. Technique of focusing - concentrating;

5. Technique of hinting;

6. Technique of development management;
7. Technique of coincidence.

\section{Introducing TRIZ Method into Edu- cational System}

Consequences of the direct increase of creativity in educational institutions imply removal of obstacles under the label "doubt" and "nih" ("not invented here"), and usage of the educational institution's own resources on the occasion of allocating key people in time. Justification of the process of introducing TRIZ method is represented by a flow chart, Figure 2, every step of which will be the subject of analysis regarding the link between direct results - new ideas, new concepts, creativity and improvement of the organizational changes' results.

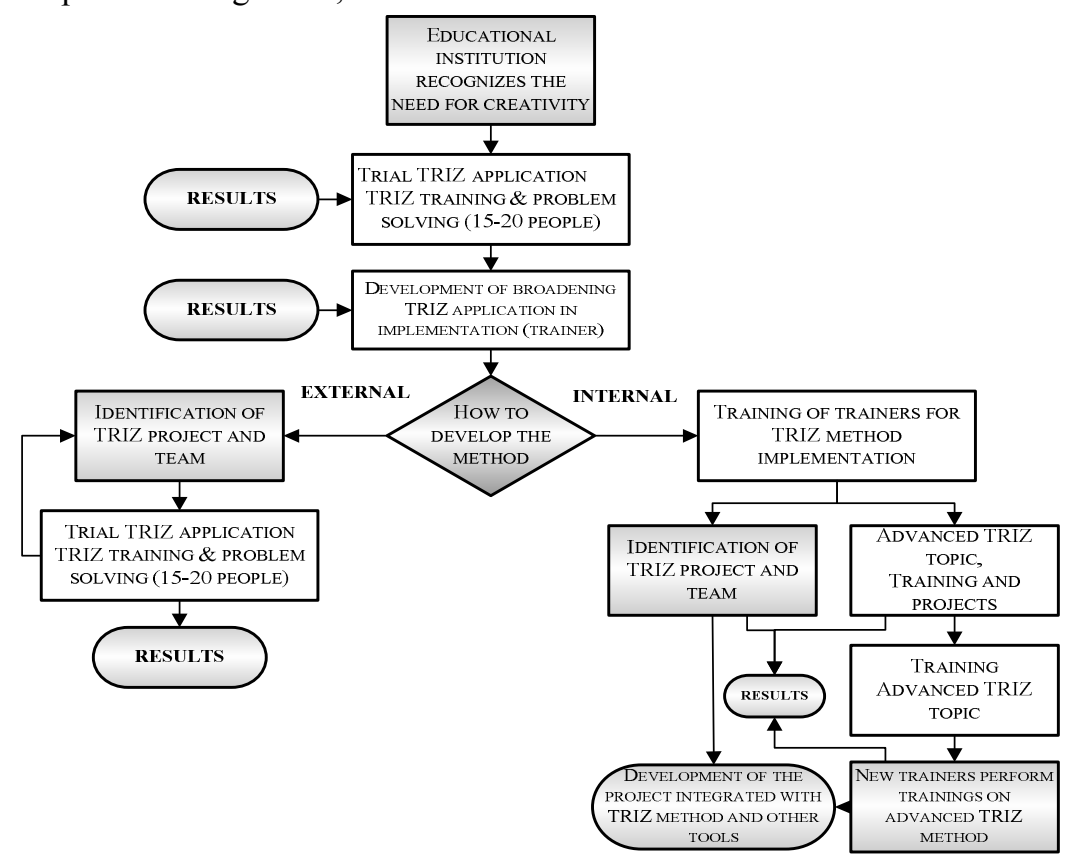

Figure 2. TRIZ implementation flow chart.

Step 1 represents the decision in educational institutions by which it is announced that increase of creativity is required. This step is caused by the need for using creative ideas, although it may come from legal regulatory requirements as well. The so called TRIZ "leaders" are selected from one or more educational institutions in the course of conducting the TRIZ method introducing and institutionalizing. Step 2 stands for selecting of one or more pilot projects for introducing the TRIZ method. Legal requirements or problems identification in the institution represent the system selection corrective actions, so that the "leader" works with consultants in selecting those problems that will have the optimum usability of future education plans. Step 3 stands for implementation of results. In accordance with the educational institution's needs, this can be done by joint efforts, by the educational institution itself. The results represent generalized solutions for the problems in implementing the concepts obtained in step 2, so that it is obvious that participants have obtained back up during work at step 2. Upon reaching success in steps 2 and 3, TRIZ "leaders" of the educational institution are grouped along one of the two paths. By an internal proceeding, a team of trained TRIZ participants from the educational institution is formed - the internal team - which will replace the consultants and obtain higher degree of experience. Hybrid approaches have been successfully used lately, where quickly obtained results of the method application are followed up, while continuity of future development is obtained by an internal procedure. Outside procedure is also often used for strategic planning, for applications in the TRIZ method, for technologies of forecasting for the educational institution, while the internal procedure is used for the development platform product. Internal experts study the strategic benefit of the TRIZ method and they become 
the internal consultants, as well as instructors, in the course of advanced themes of the training. Internal experts become collective "leaders" in overcoming the last obstacles in implementing the TRIZ method, following the right hand "internal" branch of the flow chart [4].

The TRIZ method's author identified forty characteristics - phenomena and linked the factors by creating the below stated models of technical contradictions, Table 1 . The presented table of the innovative contradictions' characte- ristics represents also the mutual actions between these events / characteristics. This method takes into consideration the influences of innovative features, Table 2 , for each of the problems identified in the normalized system. The normalized system represents a system performing certain function with the input data in the form of information, while each subsequent information input into the educational system represents a parallel function.

Table 1. The matrix of contradictions in education.( partial view).

\begin{tabular}{|c|c|c|c|c|c|c|c|c|c|c|c|c|c|c|c|c|c|c|c|c|c|}
\hline & $\begin{array}{l}\text { The feature } \\
\text { that is deteriorating } \\
\text { The feature } \\
\text { that is improving }\end{array}$ & 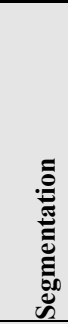 & 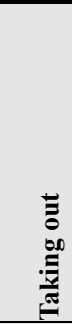 & 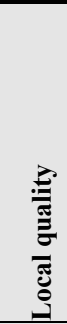 & 苞 & 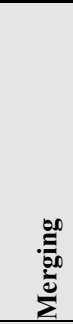 & 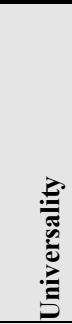 & 些 & 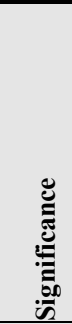 & 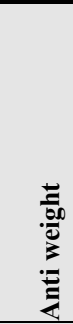 & 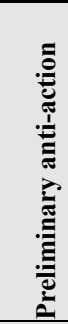 & 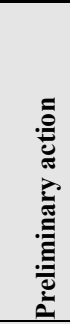 & : & 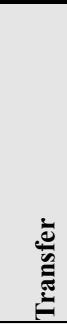 & 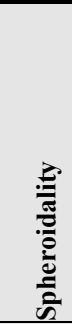 & 异 & 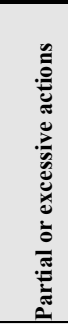 & 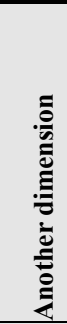 & 导 & 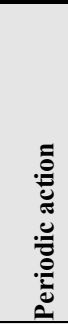 & 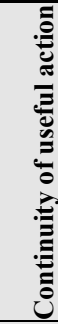 \\
\hline & & 1 & 2 & 3 & 4 & 5 & 6 & 7 & 8 & 9 & 10 & 11 & 12 & 13 & 14 & 15 & 16 & 17 & 18 & 19 & 20 \\
\hline 1 & Seomentation & & & 158 & & 2917 & & 292 & & 28 & 810 & 1036 & 1014 & 135 & 2827 & 534 & & 629 & 191 & 3512 & \\
\hline 1 & segmentation & & & 2934 & & 3834 & & 4028 & & 1538 & 1837 & 3740 & 3540 & 1939 & 1840 & 3135 & & 438 & 32 & 3431 & \\
\hline & & & & & 101 & & 3530 & & 535 & & 810 & 1329 & 1310 & 2639 & 282 & & 227 & 2819 & 1932 & & 1819 \\
\hline 2 & Taking out & - & & - & 2935 & - & 132 & - & 142 & - & 1935 & 1018 & 2914 & 140 & 1027 & - & 196 & 3222 & 35 & - & 281 \\
\hline 3 & Local quality & $\begin{array}{l}815 \\
2934\end{array}$ & - & & - & $\begin{array}{l}1517 \\
4\end{array}$ & - & $\begin{array}{l}717 \\
435\end{array}$ & - & $\begin{array}{l}134 \\
8\end{array}$ & 1710 & $\begin{array}{l}18 \\
35\end{array}$ & $\begin{array}{l}18 \\
1029\end{array}$ & $\begin{array}{l}18 \\
1534\end{array}$ & $\begin{array}{l}835 \\
2934\end{array}$ & 19 & - & $\begin{array}{l}1015 \\
19\end{array}$ & 32 & $\begin{array}{l}835 \\
24\end{array}$ & - \\
\hline 4 & Asymmetry & - & $\begin{array}{l}3528 \\
4029\end{array}$ & - & & - & $\begin{array}{l}177 \\
1040\end{array}$ & - & $\begin{array}{l}358 \\
214\end{array}$ & - & 2810 & $\begin{array}{l}114 \\
35\end{array}$ & $\begin{array}{l}1314 \\
157\end{array}$ & $\begin{array}{l}3937 \\
35\end{array}$ & $\begin{array}{l}1514 \\
2826\end{array}$ & - & $\begin{array}{l}110 \\
35\end{array}$ & $\begin{array}{l}335 \\
3818\end{array}$ & 325 & - & - \\
\hline 5 & Merging & $\begin{array}{l}217 \\
294\end{array}$ & . & $\begin{array}{l}1415 \\
184\end{array}$ & - & & - & $\begin{array}{l}714 \\
174\end{array}$ & - & $\begin{array}{l}2930 \\
434\end{array}$ & $\begin{array}{l}1930 \\
352\end{array}$ & $\begin{array}{l}1015 \\
3628\end{array}$ & $\begin{array}{l}534 \\
294\end{array}$ & $\begin{array}{l}112 \\
1339\end{array}$ & $\begin{array}{l}315 \\
4014\end{array}$ & 63 & - & $\begin{array}{l}215 \\
16\end{array}$ & $\begin{array}{l}1532 \\
1913\end{array}$ & 1932 & - \\
\hline 6 & Universality & - & $\begin{array}{l}302 \\
1418\end{array}$ & - & $\begin{array}{l}267 \\
939\end{array}$ & - & & - & - & - & $\begin{array}{l}118 \\
3536\end{array}$ & $\begin{array}{l}1015 \\
3637\end{array}$ & - & 238 & 40 & - & $\begin{array}{l}210 \\
1930\end{array}$ & $\begin{array}{l}3539 \\
38\end{array}$ & - & - & - \\
\hline 7 & Grouping & $\begin{array}{l}226 \\
2940\end{array}$ & - & $\begin{array}{l}17 \\
435\end{array}$ & - & $\begin{array}{l}17 \\
417\end{array}$ & - & & - & $\begin{array}{l}294 \\
3834\end{array}$ & $\begin{array}{l}1535 \\
3637\end{array}$ & $\begin{array}{l}635 \\
3637\end{array}$ & $\begin{array}{l}115 \\
294\end{array}$ & $\begin{array}{l}2810 \\
139\end{array}$ & $\begin{array}{l}914 \\
157\end{array}$ & $\begin{array}{l}635 \\
4\end{array}$ & - & $\begin{array}{l}3439 \\
1018\end{array}$ & $\begin{array}{l}213 \\
10\end{array}$ & 35 & - \\
\hline 8 & Significance & - & $\begin{array}{l}3510 \\
1914\end{array}$ & 1914 & $\begin{array}{l}358 \\
214\end{array}$ & - & - & - & & - & $\begin{array}{l}218 \\
37\end{array}$ & 2435 & $\begin{array}{l}72 \\
35\end{array}$ & $\begin{array}{l}3428 \\
3540\end{array}$ & $\begin{array}{l}914 \\
1715\end{array}$ & - & $\begin{array}{l}3534 \\
38\end{array}$ & $\begin{array}{l}356 \\
4\end{array}$ & - & - & - \\
\hline 9 & Anti weight & $\begin{array}{l}228 \\
1338\end{array}$ & - & $\begin{array}{l}1314 \\
8\end{array}$ & - & $\begin{array}{l}2930 \\
34\end{array}$ & - & $\begin{array}{l}729 \\
34\end{array}$ & - & & $\begin{array}{l}1328 \\
1519\end{array}$ & $\begin{array}{l}618 \\
3840\end{array}$ & $\begin{array}{l}3515 \\
1834\end{array}$ & $\begin{array}{l}2833 \\
118\end{array}$ & $\begin{array}{l}83 \\
2614\end{array}$ & $\begin{array}{l}319 \\
355\end{array}$ & - & $\begin{array}{l}2830 \\
362\end{array}$ & $\begin{array}{l}1013 \\
19\end{array}$ & $\begin{array}{l}815 \\
3538\end{array}$ & - \\
\hline 10 & $\begin{array}{l}\text { Preliminary } \\
\text { anti-action }\end{array}$ & 3718 & 1813 & 1719 & 2810 & 1910 & 3637 & 1237 & 1837 & 1328 & & 1821 & 1035 & 3510 & 3510 & 192 & - & 3510 & - & $\begin{array}{l}1917 \\
10\end{array}$ & 116 \\
\hline 11 & Preliminary action & $\begin{array}{l}1036 \\
3740\end{array}$ & $\begin{array}{l}1329 \\
1018\end{array}$ & $\begin{array}{l}3510 \\
36\end{array}$ & $\begin{array}{l}351 \\
1416\end{array}$ & $\begin{array}{l}1015 \\
3628\end{array}$ & $\begin{array}{l}1015 \\
3637\end{array}$ & $\begin{array}{l}635 \\
10\end{array}$ & 3524 & $\begin{array}{l}635 \\
36\end{array}$ & $\begin{array}{l}3635 \\
21\end{array}$ & & $\begin{array}{l}354 \\
1510\end{array}$ & $\begin{array}{l}3533 \\
240\end{array}$ & $\begin{array}{l}918 \\
340\end{array}$ & $\begin{array}{l}193 \\
27\end{array}$ & - & $\begin{array}{l}3539 \\
192\end{array}$ & - & $\begin{array}{l}1424 \\
1037\end{array}$ & - \\
\hline 12 & Equipotentiality & $\begin{array}{l}810 \\
2940\end{array}$ & $\begin{array}{l}1510 \\
263\end{array}$ & $\begin{array}{l}2934 \\
54\end{array}$ & $\begin{array}{l}1314 \\
107\end{array}$ & $\begin{array}{l}534 \\
410\end{array}$ & - & $\begin{array}{l}144 \\
1522\end{array}$ & $\begin{array}{l}72 \\
35\end{array}$ & $\begin{array}{l}3515 \\
3418\end{array}$ & $\begin{array}{l}3510 \\
3740\end{array}$ & $\begin{array}{l}3415 \\
1014\end{array}$ & & $\begin{array}{l}331 \\
184\end{array}$ & $\begin{array}{l}3014 \\
1040\end{array}$ & $\begin{array}{l}1426 \\
925\end{array}$ & - & $\begin{array}{l}2214 \\
1932\end{array}$ & $\begin{array}{l}1315 \\
32\end{array}$ & $\begin{array}{l}26 \\
3414\end{array}$ & - \\
\hline 13 & Transfer & $\begin{array}{l}2135 \\
239\end{array}$ & $\begin{array}{l}2639 \\
140\end{array}$ & $\begin{array}{l}1315 \\
128\end{array}$ & 37 & $\begin{array}{l}211 \\
13\end{array}$ & 39 & $\begin{array}{l}2810 \\
1939\end{array}$ & $\begin{array}{l}3428 \\
3540\end{array}$ & $\begin{array}{l}3315 \\
2818\end{array}$ & $\begin{array}{l}1035 \\
2116\end{array}$ & $\begin{array}{l}235 \\
40\end{array}$ & $\begin{array}{l}221 \\
184\end{array}$ & & $\begin{array}{l}179 \\
15\end{array}$ & $\begin{array}{l}1327 \\
1035\end{array}$ & $\begin{array}{l}393 \\
3523\end{array}$ & $\begin{array}{l}351 \\
32\end{array}$ & $\begin{array}{l}323 \\
2716\end{array}$ & 1319 & $\begin{array}{l}274 \\
2918\end{array}$ \\
\hline 14 & Spheroidality & $\begin{array}{l}18 \\
4015\end{array}$ & $\begin{array}{l}4026 \\
271\end{array}$ & $\begin{array}{l}115 \\
835\end{array}$ & $\begin{array}{l}1514 \\
2826\end{array}$ & $\begin{array}{l}334 \\
4029\end{array}$ & $\begin{array}{l}940 \\
28\end{array}$ & $\begin{array}{l}1015 \\
147\end{array}$ & $\begin{array}{l}914 \\
1715\end{array}$ & $\begin{array}{l}813 \\
2614\end{array}$ & $\begin{array}{l}1018 \\
314\end{array}$ & $\begin{array}{l}103 \\
1840\end{array}$ & $\begin{array}{l}1030 \\
3540\end{array}$ & $\begin{array}{l}1317 \\
35\end{array}$ & & $\begin{array}{l}273 \\
26\end{array}$ & - & $\begin{array}{l}3010 \\
40\end{array}$ & 3519 & $\begin{array}{l}1935 \\
10\end{array}$ & 35 \\
\hline 15 & Dynamics & $\begin{array}{l}195 \\
3431\end{array}$ & - & $\begin{array}{l}219 \\
9\end{array}$ & - & $\begin{array}{l}317 \\
19\end{array}$ & - & $\begin{array}{l}102 \\
1930\end{array}$ & - & 335 & 192 & 193 & $\begin{array}{l}1426 \\
2825\end{array}$ & 133 & $\begin{array}{l}273 \\
10\end{array}$ & & - & $\begin{array}{l}1935 \\
39\end{array}$ & $\begin{array}{l}219 \\
435\end{array}$ & $\begin{array}{l}286 \\
3518\end{array}$ & - \\
\hline 16 & $\begin{array}{l}\text { Partial or } \\
\text { excessive actions }\end{array}$ & - & $\begin{array}{l}627 \\
1916\end{array}$ & - & $\begin{array}{l}140 \\
35\end{array}$ & - & - & - & $\begin{array}{l}3534 \\
38\end{array}$ & - & - & - & - & 3523 & - & - & & $\begin{array}{l}1918 \\
3640\end{array}$ & - & - & - \\
\hline 17 & Transfer & $\begin{array}{l}3622 \\
638\end{array}$ & $\begin{array}{l}2235 \\
32\end{array}$ & $\begin{array}{l}1519 \\
9\end{array}$ & $\begin{array}{l}1519 \\
9\end{array}$ & $\begin{array}{l}335 \\
3918\end{array}$ & 3538 & $\begin{array}{l}3439 \\
4018\end{array}$ & $\begin{array}{l}356 \\
4\end{array}$ & $\begin{array}{l}228 \\
3630\end{array}$ & $\begin{array}{l}3510 \\
321\end{array}$ & $\begin{array}{l}3539 \\
192\end{array}$ & $\begin{array}{l}1422 \\
1932\end{array}$ & $\begin{array}{l}135 \\
32\end{array}$ & $\begin{array}{l}1030 \\
2240\end{array}$ & $\begin{array}{l}1913 \\
39\end{array}$ & $\begin{array}{l}1918 \\
3640\end{array}$ & & $\begin{array}{l}3230 \\
2116\end{array}$ & $\begin{array}{l}1915 \\
317\end{array}$ & - \\
\hline 18 & Frequency & $\begin{array}{l}191 \\
32\end{array}$ & $\begin{array}{l}235 \\
32\end{array}$ & $\begin{array}{l}1932 \\
16\end{array}$ & - & $\begin{array}{l}1932 \\
26\end{array}$ & - & $\begin{array}{l}213 \\
10\end{array}$ & - & $\begin{array}{l}1013 \\
19\end{array}$ & $\begin{array}{l}2619 \\
6\end{array}$ & - & 3230 & $\begin{array}{l}323 \\
27\end{array}$ & 3519 & $\begin{array}{l}219 \\
6\end{array}$ & - & $\begin{array}{l}3235 \\
19\end{array}$ & & $\begin{array}{l}321 \\
19\end{array}$ & $\begin{array}{l}3235 \\
115\end{array}$ \\
\hline 19 & Periodic action & $\begin{array}{l}1218 \\
2831\end{array}$ & - & 1228 & - & $\begin{array}{l}1519 \\
25\end{array}$ & $\cdot$ & $\begin{array}{l}3513 \\
18\end{array}$ & 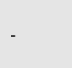 & $\begin{array}{l}835 \\
35\end{array}$ & $\begin{array}{l}1626 \\
212\end{array}$ & $\begin{array}{l}2314 \\
25\end{array}$ & $\begin{array}{l}122 \\
29\end{array}$ & $\begin{array}{l}1913 \\
1724\end{array}$ & $\begin{array}{l}519 \\
935\end{array}$ & $\begin{array}{l}2835 \\
618\end{array}$ & 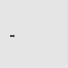 & $\begin{array}{l}1924 \\
314\end{array}$ & $\begin{array}{l}215 \\
19\end{array}$ & & - \\
\hline 20 & Continuity of & - & 199 & - & - & - & - & - & - & - & 3637 & - & - & 274 & 35 & - & - & - & 192 & - & \\
\hline
\end{tabular}


Table 2. Innovative principles in education [6].

\begin{tabular}{|c|c|c|}
\hline TRIZ Principle & $\begin{array}{l}\text { Innovative principle } \\
\text { description }\end{array}$ & $\begin{array}{l}\text { Actions which enable redefining teaching activities by interpreting business } \\
\text { functions, educational concepts and terms }\end{array}$ \\
\hline \multirow{5}{*}{$\begin{array}{l}\text { (17) } \\
\text { Transition to } \\
\text { other dimensions }\end{array}$} & $\begin{array}{l}\text { a. Transfer the event into two- } \\
\text { or three-dimensional space }\end{array}$ & $\begin{array}{l}\text { Offer teaching programming available by Internet or computer networking, with instructions } \\
\text { received at locations outside classrooms supervised by the physical presence of teachers. } \\
\text { Celebrate special events of other cultures. } \\
\text { Use more experimental than theoretical learning. } \\
\text { Use the knowledge of guest lecturers. }\end{array}$ \\
\hline & $\begin{array}{l}\text { b. Consider usage of } \\
\text { dimensions or movement } \\
\text { outside current plane. }\end{array}$ & $\begin{array}{l}\text { Use multidimensional satisfaction of parents by taking polls. } \\
\text { Open the capacities during evenings or weekends. } \\
\text { Make internal communication line and virtual support after the school day. } \\
\text { Change the teaching by way of various styles of learning. }\end{array}$ \\
\hline & $\begin{array}{l}\text { c. Use multi-storey instead } \\
\text { of single storey event }\end{array}$ & $\begin{array}{l}\text { Make the difference and divide pupils on the basis of their needs, behavior, age, etc. } \\
\text { Use cooperative learning and constructivism. } \\
\text { Use multiple examples and practical opportunities. }\end{array}$ \\
\hline & $\begin{array}{l}\text { d. Direct disputes towards } \\
\text { the event which is on the } \\
\text { right side. }\end{array}$ & $\begin{array}{l}\text { Make levels according to grades, or interdisciplinary teams. Allocate to the classroom the in- } \\
\text { stitution's educational responsibilities. } \\
\text { Support horizontal communication between teachers. } \\
\text { Draft a mobile library concept. }\end{array}$ \\
\hline & $\begin{array}{l}\text { e. Use the "third party", } \\
\text { in the given area. }\end{array}$ & $\begin{array}{l}\text { Divide and interpret pupils' achievement results in the field of learning, in fields and plans, } \\
\text { improve work with parents, school council and other interested groups. } \\
\text { Show assessments of other schools according to fields, using outside consultants for the pro- } \\
\text { gram or school. } \\
\text { Establish two-way communication between teachers, parents, doctors, etc. } \\
\text { Coordinate usage of facilities between school and social needs. } \\
\text { Use tutor's knowledge and assessments at the level of compeers. }\end{array}$ \\
\hline
\end{tabular}

\section{Conditions for Contradiction Matrix Application}

TRIZ method is based on applying the principle of contradiction, i.e. the 40 principles (Table 1), which are capable of fulfilling all expectations, [2] only if the following laws are applied:

\subsection{Law on Ideal System Development}

Ideal system is defined as the best possible solution in given contradictions, when there are no limitations of resources.

$$
\text { Ideality }=\Sigma \text { Benefits } / \text { Costs }+\Sigma \text { Harms. }
$$

\subsection{Law of Unity or Joint Penetration and Contradictions}

Technical development occurs by reciprocal actions of contradictions. The philosophical Yin and Yang have to communicate and to overcome mutual contradictions.

\subsection{Law on Negating Negation}

Development consists of reiterating the same steps, continually, but on a higher development level by way of more advanced technologies, the set of experience and elements. Currently, the educational system model has got an asymmetric structure between legislation institutions - the Ministry of education and educational institutions, with the tendency towards the existence of symmetry, so that the results of basic values in future will be in the area of - consulting partnership.

\subsection{Law on Transforming Quantity into Quality}

Under influence of the time function, quantitative changes pass into certain qualitative ones.

\subsection{Law on Uneven Sub-System Development}

Various portions of the educational system have developed in uneven steps.

\subsection{Law on System Transition to Higher Levels}

Systems develop from mono- or single-system functions into two- or multi-functional systems. In the course of educational institutions developing, expert associates and lec- 
turers will be invited to analyze the market needs, as well as to design education model, perform original research works, provide consulting functions and to follow up realization "from end-to-end", i.e. from the start of education till "delivery" of creative experts.

\subsection{Law on Transfer Form Macro Level to Micro Level}

Educational systems develop according to development of their respective sub-systems. Various micro-competency centers will be developed, with each of them directed to the segment of "market" surroundings, with the tendencies of increasing knowledge bases.

\section{TRIZ Method Support in Education}

Bearing in mind the fact that TRIZ method is based on 7 pillars, Figure 3, their possible significance is stated for support to the system's educational function.
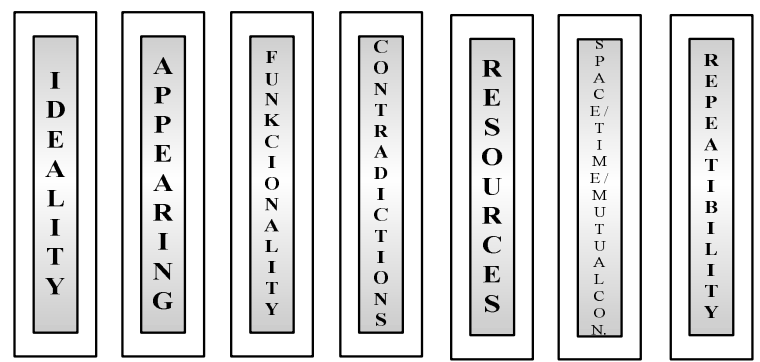

Figure 3. Law of seven TRIZ model pillars.

IDEALITY: Any successful creativity is developed in the direction towards increasing ideality - more benefits, less costs, less harm. In generating and maintaining creative individuals, development interruptions can be viewed as the concept of "only/self-X"??, so that education which "writes itself" is achieved by accomplishing goals through numerous teachers' activities.

APPEARING: This pillar has been introduced into the TRIZ method so that relevant business situations could be envisaged by the method in the process of education.

FUNCTIONALITY: Educational system should be focused on generating creativity function and on services provided, and not only on generating creativity among students.

CONTRADICTIONS: Educational systems are developed in the direction of increasing ideality through consecutive dangers and solving conflicts and contradictions. Contradictions - eliminate the strategies planned by others, and they can be observed as the primary instigator of creativity development.

RESOURCES: Anything in educational system that has not been used to its maximum represents a potential resource of the educational institution.

SPACE / TIME / MUTUAL CONNECTION: Human brain is susceptible to the outcome known under the name of psychological inertia. Transposed consideration perspective includes physical space, time-based decision or the way in which various educational system elements are mutually linked and relate to one another.

REPEATABILITY: This pillar does not belong to TRIZ method in the classical sense, but it can be found in the "professional" TRIZ method.

\section{Levels of Creativity}

Implementation of the TRIZ method can be conducted independently from the level of creativity. Creativity levels are discrete innovation levels at which events occur [1]. In the course of research, the following levels of research were observed, describing the process analyzed at "micro centers" (group of secondary school network):

a) LEVEL 1: Routine education solves problems by the methods known within the specialty.

b) LEVEL 2: Slight educational system improvements, by using known methods, such as $6 \Sigma$, Lean, etc.

c) LEVEL 3: Improvement of the existing educational system basis by way of methods known outside the educational institution.

d) LEVEL 4: New generation educational systems imply new principles for performing the primary educational system's functions.

e) LEVEL 5: Scientific discoveries leading to fundamental advancements are rare.

\section{Innovative Principles in Education}

Contradiction matrix in education, Table 1 [6], and the forty innovative principles has also found application in the field of social human activities. Efforts are made to find a potential solution of the concept generating experiences and knowledge in education by way of the contradiction matrix, according to which this paper represents a theoretical contribution to solving conflicts in the field of education. The descriptions of forty innovative principles and relevant actions have enabled redefining of teaching activities in the field of education, by interpreting educational functions, educational concepts and the terms acquired in the course of the analysis. In the stated example, innovative solutions-actions provide references to various levels of competences in the field of the educational institution's administration, in organizing classroom teaching, or to both areas [6]. The contradiction matrix, Table 1, shows mutual influences of contradictory features in educational systems and the innovative principles in education.

In the contradiction matrix, the fields denoted by (-) indicate that TRIZ principles do not exert mutual influences; for example, universality does not affect the feature of segmentation, homogeneity does not affect asymmetry, etc.

Mutual influences of TRIZ principles is expressed so that in certain fields of the matrix up to four digits can be found, or a matrix field is void when improvement of one TRIZ feature does not affect deterioration of any other TRIZ feature. The contradiction feature shows description of TRIZ principles and of actions necessary for raising the 
educational system's efficiency. Due to lack of space, this paper presents only the selected principle -17 Transition to other dimensions, Table 2, with description of problem solution searching and of actions which are to be taken.

\section{Four Most Influential Features' Ma- trix}

The essence in considering the contradictions matrix's effects on finding the solution of a problem, explains that the mechanisms in the essence of features are very closely connected with the features in the contradiction matrix, in the field of idealization. The methodology named four most influential matrixes has been developed with the aim of decreasing effects of participants' features on educational system quality assessment in the educational process, which represents the essence of the process of education management. Thus, progress has been made in application onto the educational system model and generating new categorization of the contradictions matrix basic principles on action, objects, duration, features, quantities, frequency, and complexity of information movement in the educational system and sequence, Table 3. By categorizing of 5 segments - the activities of dividing, transfer, change, addition and usage of other possibilities - the contradiction matrix fundamental mechanisms is represented.

Table 3. Educational system features, mechanisms and principles.

\begin{tabular}{|c|c|}
\hline \multicolumn{2}{|l|}{ ACTION } \\
\hline 1.Separate & Instead of continual action, interaction is used. \\
\hline 2.Transfer & Transfer the object or its environment. \\
\hline 3.Change & Change the dimensions into $2 \mathrm{D}$ or $3 \mathrm{D}$, the structured nature of movement is to be changed into non-structured. \\
\hline 4.Add & Add the exchangeable parts, eliminate delay, anti-action effect. \\
\hline \multicolumn{2}{|l|}{ OBJECT } \\
\hline 1. Separate & Dividing the system into segments/objects into several independent parts. \\
\hline 4. Add & Add or use the common group effect. \\
\hline \multicolumn{2}{|c|}{ DURATION } \\
\hline 1. Separate & Replace one cheap object by several cheaper objects. \\
\hline 4. Add & Add phases to processes. \\
\hline \multicolumn{2}{|c|}{ FEATURES } \\
\hline 3. Change & Change the module flexibility degree. \\
\hline \multicolumn{2}{|c|}{ QUANTITY } \\
\hline 3. Change & Increase number of parts of the entity, introduce feedback and cross checking. \\
\hline \multicolumn{2}{|c|}{ FREQUENCY } \\
\hline 3. Change & Create a self-service module in performing useful accessory functions.. \\
\hline \multicolumn{2}{|c|}{ COMPLEX MOVEMENT } \\
\hline 3. Change & Change linear into circular movement, use the centrifugal properties. \\
\hline \multicolumn{2}{|c|}{ ENVIRONMENT } \\
\hline 3. Change & Change temperature, flexibility, object concentration. \\
\hline \multicolumn{2}{|c|}{ OBJECT DIMENSIONS } \\
\hline 3. Change & Instead of lines, use planes and 3D, multilevel linking of parts. \\
\hline \multicolumn{2}{|c|}{ OBJECT POSITION } \\
\hline 3. Change & Turn the module or processes "upside down" \\
\hline \multicolumn{2}{|c|}{ OBJECT SYMETRY } \\
\hline 3. Change & Change the subsystem forms; instead of symmetrical, into asymmetrical. \\
\hline \multicolumn{2}{|c|}{ ATMOSPHERE } \\
\hline 3. Change & Enrich the air in the operating environment by $\mathrm{O}_{2}$ or neutral gases. \\
\hline \multicolumn{2}{|c|}{ FUNCTION } \\
\hline 4.Add & Mutual actions with environment, use "slightly less" or "slightly more" than the method itself. \\
\hline \multicolumn{2}{|c|}{ SCHEDULE } \\
\hline 3. Change & Separate usage venue from the location, make flanking or parallel operations. \\
\hline
\end{tabular}

The matrix of four most influential features, Table 4, is a tool developed with the objective to enable the participants in the educational system to assess the educational system's quality, on the basis of forty influential features, as well as to create a dominant strategy, support the four most influential features' mechanisms, to point out favorable and try to eliminate non-favorable object features, enable generation of creativity in solving problems among students.
According to the research results [7] comprising a number of resolved cases by a representative sample, the contradiction matrix efficiency degree was $51 \%$, while the efficiency degree of the four most influential features matrix reached $79 \%$.

Due to the limited scope of this paper, only some parameters are shown, while the others are described in the References [6]. The described analysis of the innovative 
principles frequency in educational institutions is based on the research in educational institutions that exist in the educational system.

Table 4. The matrix of four most influential features [5].

\begin{tabular}{|c|c|c|c|c|}
\hline \multirow{2}{*}{$\begin{array}{l}\text { Improved } \\
\text { Matrix } \\
\text { parameters } \\
1 \text { Segmentation }\end{array}$} & \multicolumn{4}{|c|}{$\begin{array}{l}\text { Features with major influence on the educational } \\
\text { system }\end{array}$} \\
\hline & Features & Object & Environment & Atmosphere \\
\hline 2. Separability & Features & Object & Action & Duration \\
\hline 3. Local quality & Object & Features & Symmetry & Position \\
\hline 4. Asymmetry & Features & Object & $\begin{array}{l}\text { Complex } \\
\text { movement }\end{array}$ & Frequency \\
\hline 5. Linkability & Object & Dimensions & Action & Features \\
\hline 6. Universality & Frequency & Features & Frequency & Atmosphere \\
\hline 7. Grouping & Features & Object & Symmetry & Function \\
\hline 8. Significance & Features & Object & Frequency & $\begin{array}{l}\text { Complex } \\
\text { movement }\end{array}$ \\
\hline 9. Counteractions & Environmen & Features & Action & Object \\
\hline $\begin{array}{l}\text { 10.Previous } \\
\text { actions }\end{array}$ & Features & Object & Frequency & Action \\
\hline
\end{tabular}

In analyzing the principle allocation, it should be pointed out that highest upturn has been realized by the following principles: No.11- Preliminary action, No. 23- Feedback information, while the largest drop in the sequence of principles in educational system has been experienced by principles No. 18- Outside influences and No. 30- Flexibility and transparency.

According to the sequence of appearing of educational institutions' innovative principles in the contradiction matrix, it can be noticed that the innovative principle No. $10-$ Preliminary action, has been most frequently used, and then, the paper lists only the first ten most represented principles: principle No. 3 - Local quality, principle No. 24 - Intermediary, principle No. 13 - Relocation /"the other way round", principle No. 2 - Separability/Taking out, principle No. 25 - Self-service, principle No. 35 - Parameter changes, principle No. 7 - Grouping/Russian dolls, principle No. 1 - Segmentation and principle No. 4 Asymmetry. According to frequency, the last principle is No. 38 - Oxidation/Strong oxidants.

\section{Conclusion}

The paper considers application of the TRIZ method through proposing different principles of decision making and qualitative estimation. The contradictions stated in the analysis of the process of education so far, and of planning and implementation of new teaching programs into the educational system are generally solved by searching for compromise solutions. Implementation of the TRIZ method implies increase of creativity through the process of bringing compromised solutions, by including the ideas from the theories of complexity and cybernetics into the philosophy basis, by the use of contradiction matrix.

Contradictions prove, on the basis of analyzing the education field regulations and safety and experiences during educational system functioning, that creativity is more than art. It is estimated that application of the classical TRIZ method principles is becoming necessary but not sufficient if it is to become a part of a "systematic education development for a creative discussion". The TRIZ laws on educational system development can be used for predicting trends in the market and therefore they have to be ready for developing new possibilities as the part of educational plans. The results of this paper show that for the future TRIZ instructors of technological education, the very introduction of the TRIZ method in the syllabus represents in itself an award for them. It is believed that it would be suitable to introduce the TRIZ method in resolving problems in secondary/grammar schools, as the thematic content of the training. As regards the level of education at which introducing TRIZ training should be commenced, the ages of the 2 nd and 3rd secondary grades are considered as the optimum. In the Ministry of Education and Science, possibilities are considered, and feasibility studies conducted, for discovering, applying and supporting creativity. The authors have gained experience in TRIZ-education and have used numerous experiences from teaching.

The next research should follow the comparative analyses of education process management by contradiction matrix, development of the matrix of four most influential features and decision making matrix. It has been proven that in future, educational system development should be analyzed and evaluated together with those that are not of information, technical and industrial nature.

\section{References}

[1] Altshuller G. S., (1971) Теория Решеня Изобретательных Задач - ТРИЗ, Innovation Algorithm. Worcester, Massachusetts.

[2] Mann, D., Marsh, D ., Waters, F., (2002) „Using TRIZ to Resolve Educational Delivery Conflicts Inherent to Expelled Students in Pennsylvania“.

[3] Hohnjec M., Soković M. (2012): «TRIZ for Systematic Innovation», International Conention on Quality ' ICQ 2012, JUSK, International Journal „Advanced Quality“ Vol. 40, No. 2, pp 20-25, Belgrade.

[4] Kaplan L., Malkin S., Proseanic V., Visnepolschi S., Zlotin, A., Zusman A., (2001) „TRIZ beyond technology“, The theory and practice of applying TRIZ to nontechnical areas".

[5] Stefanović N., Mitrović R., Popović P., Ristivojević M. (2007): «A Contribution to Solving of Integration Contradiction Problem in Designing Engineering Systems», International Conference 'International Convention on Quality, Organized by YUSQ, Belgrade, Introduction paper, Total Quality Management and Excelence, Vol.35, No.1-2, Belgrade. 
[6] Stefanović N., (2010) Ph.D. thesis "Model risk assessment of information systems", Novi Sad, Faculty of Economy and Engineering Management.
[7] Zusman, A., (1999): „Overview of Creative Methods“, TRIZ Journal. 